\title{
Application of Electronic Load in Power Supply Test
}

\author{
Hu Wenting \\ Beijing Spacecraft, Shaanxi Province, 100094
}

Keywords: electronic load; power test; methods; aviation

\begin{abstract}
Electronic load, as a new type of electronic equipment, has been widely used in power testing, and plays a very important role in technology guidance. This paper presents a variety of electronic load power testing methods, and conducts empirical analysis of its specific technical application in aviation power testing.
\end{abstract}

Compared with the traditional manual test method, the electronic load can be automatically tested for the power supply. Compared with the traditional manual test method, it also adds the test circuit and simulation test results, which has greater advantages in technical application as a whole. In other words, the electronic load can test the power supply characteristics properly, which can simulate the electric load in the real environment and combine the constant current, constant resistance, constant voltage and constant power function mode. It can be said that the test methods of short circuit, overcurrent and dynamic mode of power supply are quite abundant.

\section{Technical Application of Voltage Source Testing with Electronic Load}

The traditional testing method for voltage source is manual test. In the process of testing, the load resistance value must be adjusted frequently. The measuring process is not simple enough, and the measurement error is easy to occur. In the process of manual voltage source testing, it involves the test of open circuit voltage of power supply EMF, internal resistance of power supply and so on. Since there is only one equivalent resistance and one load resistance in the open circuit voltage, it is necessary to adjust the measurement manually to obtain accurate results. The specific test method is to first change the value of the load resistance to ensure that the ammeter can normally measure the current, then measure the output voltage through the voltmeter, and finally record all the measured open circuit voltage data.

Compared with manual testing, the electronic load test proposed in this paper is more convenient. It uses a constant current electronic load that sets the current, as shown in figure 1.

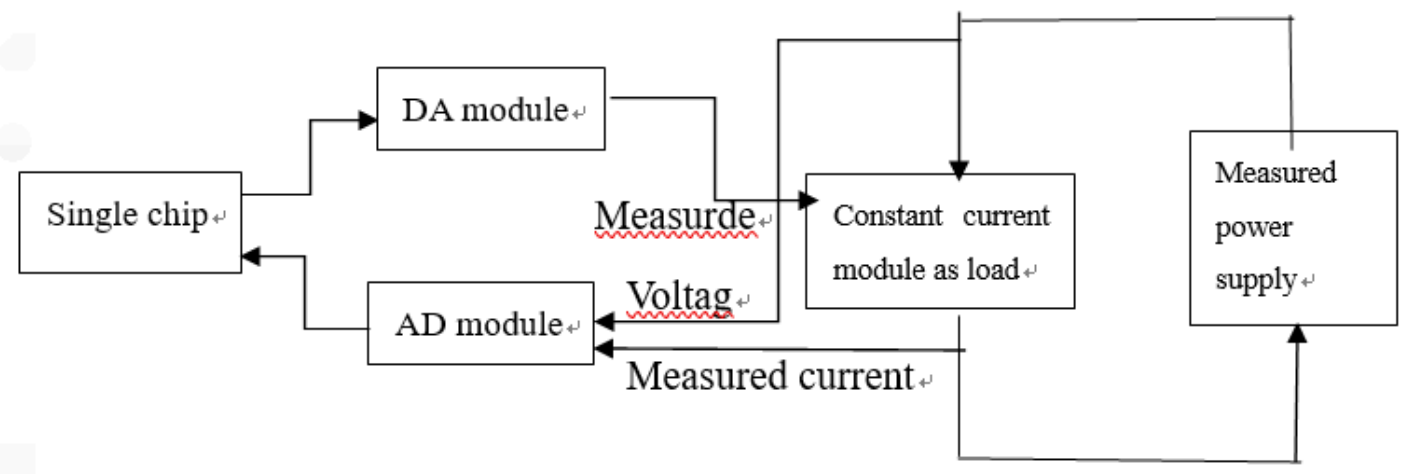

Figure 1 Technical flowchart of voltage source testing using constant current electronic load

The power supply test method mainly conforms to the technical requirements proposed by the single chip microcomputer, outputs the digital quantity accurately, tests the current of the constant current electronic load module controlled by the DA module, and understands the specific status of the measured current and voltage. It includes the detailed data record when they are read into the single chip computer. After all the automatic tests have been completed, a complete set of 
simulation circuits can be generated, as shown in figure 2 .

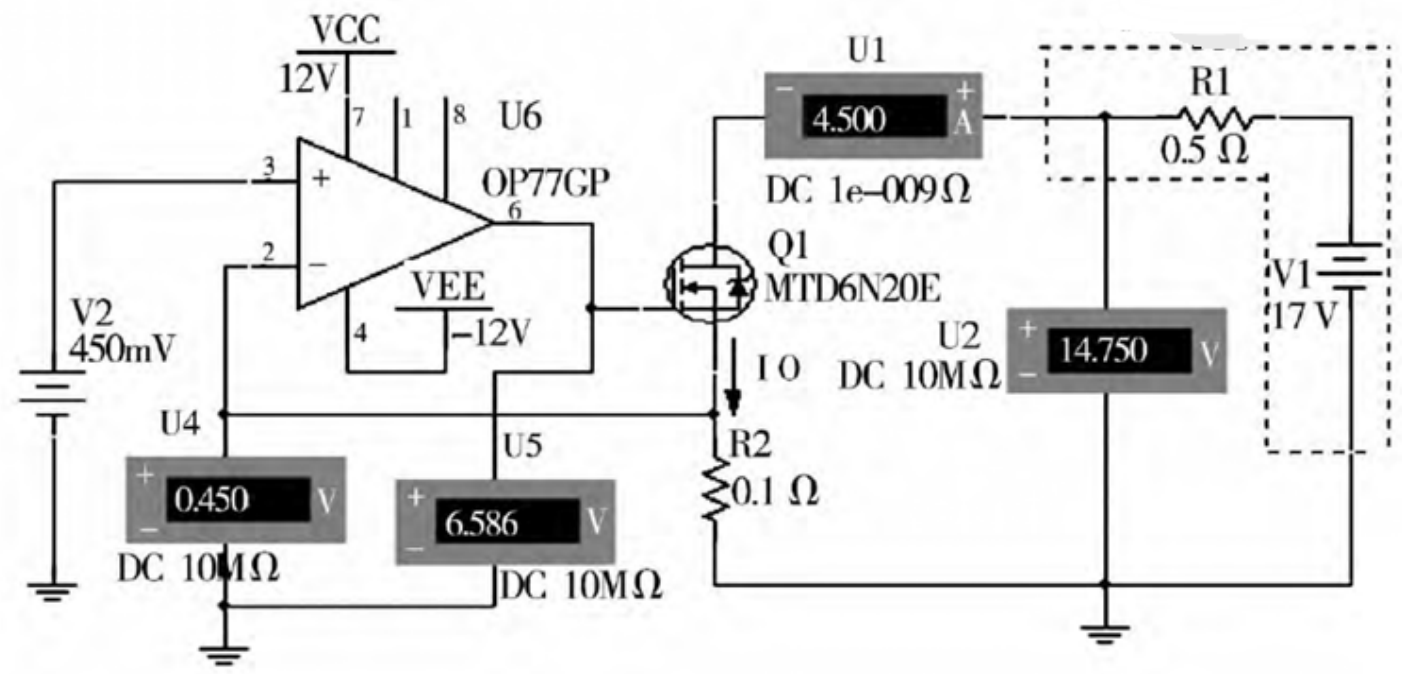

Fig. 2 Simulation circuit diagram of voltage source test using constant current electronic load

As shown in figure 2 above, the output digital quantity is specially set according to the voltage source test requirement. Finally, the effective control process of the constant current electronic load module is realized through DA module. It is guaranteed that its normal working state can be carried out in the specified current state, which is also considered to be the measured current. At the same time, the measured voltage value will be read into the MCU through AD conversion module and recorded, so as to complete the subsequent automatic test part of the voltage source to form a simulation circuit. In figure 2, the position of the dashed frame represents the voltage source being measured, and its field effect is primarily responsible for managing the $\mathrm{Q}_{1}$ power load. This setting replaces the load resistance $R_{2}$ existing in the traditional manual test, and the output current of other power sources is $I_{O}$. The resistor $R_{2}$ can be sampled here and then the negative feedback of current can be introduced to achieve the effect of changing the value of $\mathrm{V}_{2}$ and the value of load current $\mathrm{I}_{\mathrm{O}}$. It can be concluded that $\mathrm{I}_{\mathrm{O}}$ is:

$$
I_{O}=\frac{V_{2}}{R_{2}}
$$

The load current of the $\mathrm{Q}_{1}$ power supply load part can be calculated by bringing in the data. According to the power supply test method, the voltage source electronic load data can be effectively obtained, and the obtained data is relatively intact, which can meet all the conditions of automatic implementation of the test [1].

\section{Application of Electronic Load in Testing Current Source}

The constant voltage electronic load equipment described above can also be used to test the current source, that is, to test the power supply of the current mode power supply. The current source of the power supply circuit of the LED lamp is used in this paper. The LED lamp itself is quite strict with the current value of the power supply, but it does not have much technical specification for the supply voltage. Generally, when the output current of current source in LED lamp is constant current, its load resistance will change, and the output voltage will also change at the same time. So in the process of testing the current source of LED and so on, we can also use this kind of constant voltage electronic load equipment to test the relation curve between output voltage and output current.

In the actual testing process, the change of the output voltage should be determined during the load change of the current source, and the output current should be reduced to the best state. Then the simulation circuit of the current source under the background of electronic load testing can be given. In the process of constant voltage electronic load testing, a voltage negative feedback circuit 
is specially formed. The load voltage can be measured according to the voltage divider or the in-phase input $\mathrm{U}+$. Using MCU and R2 current sampling resistance to join together to build the protection circuit, as shown in figure 3.

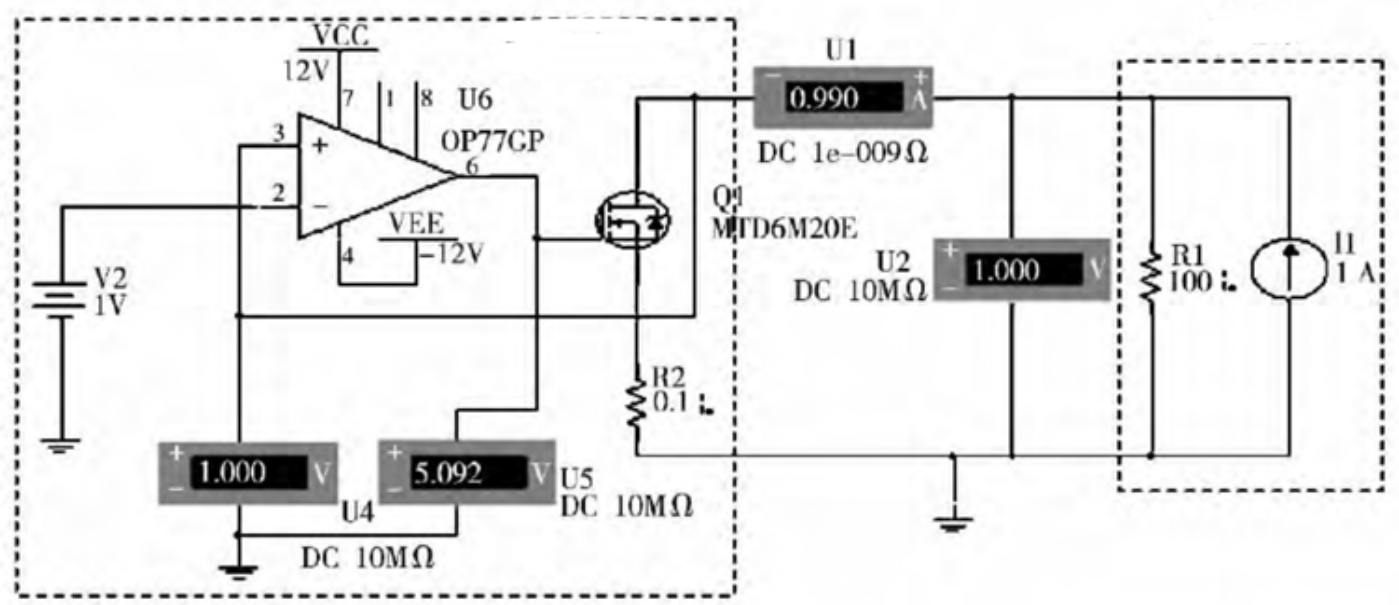

Fig. 3 schematic diagram of current source test using constant voltage electronic load equipment

Build the protection circuit based on the input, voltage, and so on in figure 3, as follows:

$$
\begin{aligned}
& U+=U_{O}, U-=V_{2} \\
& U+=U- \\
& U_{O}=V_{2}
\end{aligned}
$$

In the process of testing the current source, it is necessary to set the voltage value of the electronic load on the constant voltage electronic load first, then get the output current value accurately, and finally obtain the series voltage value. Based on the above data, the load characteristic curve of current source is drawn, and the specific value of current source internal resistance is calculated. The internal resistance $r_{O}$ should be [2]:

$$
r_{O}=\frac{\Delta U_{O}}{\Delta I_{O}}
$$

\section{Case Analysis}

At present, it is possible to test aero-generators with electronic load equipment, and it will also be applied to computer technology and automatic control technology, which combine with electronic load to form a kind of integrated power electronic device. Its rear inverter side can form a photovoltaic grid-connected device with grid-connected, and meet the unity of the functional configuration of the device. The aeronautical generator which is built on the electronic load equipment can simulate the common load characteristics, and the function application is quite convenient, which will not cause energy waste and other adverse phenomena, so it has high practical value and market development prospects. This paper hopes to use the energy-fed electronic load equipment to test the AC output power supply of the aero-generator and ensure that the aero-generator can be constructed by simulation software at different frequencies. The system then is verified by simulation to prove that the application of electronic load equipment in aero-generator power supply test is superior.

\subsection{Design of system control strategy for aero generator power supply}

The traditional aero-generator power supply mainly uses dual PWM converter to feed the electronic load, which needs to clear the mainstream topology infrastructure, and effectively maintain the three-phase power network balance to ensure that the equipment in the standard power and capacity background three-phase power network. Nowadays the three-phase energy fed electronic load can effectively improve the three-phase grid-connected structure in the circuit 
topology, which is a further upgrade of the traditional three-phase grid balancing technology.

Firstly based on the basic principle of PWM rectifier, the inverter part of aero generator in power supply is conducted with voltage output to meet the basic conditions of voltage modulation, then by controlling the AC side voltage modulation part, the effective control of grid connected current and voltage vector is ensured, which is to ensure the phase of the difference between the two in less than $180^{\circ}$, so the circuit can work in power factor always stay in the -1 state. At the same time, the inverter of aero-generator and equivalent circuit will also show a certain vector relationship. For example, if the PWM inverter is in the working state, it should satisfy the sum of the sub phase voltage and the total voltage, and the voltage on the inductor should be $90^{\circ}$ to the grid voltage, which satisfies the basic function requirement [3] of the output power factor as "- 1 ".

\subsection{Design of system control strategy for aero generator power supply}

The calculation method, modulation method and so on in the PWM control technology are used to cooperate with the electronic load equipment, the system control and rectifier side control strategy are implemented, the design scheme is worked out, and the current hysteresis comparison method is adopted. The design of the controlled circuit is simple, and the response speed is fast and the tracking accuracy is high. The position of the inverter side is controlled by triangle wave modulation, which ensures the traditional voltage and current to form double closed loop control.

Specifically, the load type of aero-generator power supply should be analyzed by pre-calculation method, and the mathematical expression under the background of instruction current should be obtained, and then the basic analog quantity of feedback current should be calculated according to the actual data. The error relation is obtained when the analog quantity is converted to the instruction. In this paper, the error value can be brought into the hysteresis comparator, and the high and low level logic can be generated by setting the upper and lower threshold, and then the compared high and low level logic signal can be transmitted to the unprocessed part, and the control algorithm can be solved. Finally, the logic control of each switch tube is realized based on the driving signal output of the switch tube.

In general, PI current loop is used in the traditional aero generator power supply, which has the problem of current tracking lag and may not be able to quickly eliminate the low frequency harmonic problems in the current. So we can use the current loop proportional resonant regulator to optimize the resonant link to ensure that the power supply voltage and current output frequency gain reaches the maximum level to meet the requirement of zero steady-state error. At the same time, the basic characteristics that the current loop resonant regulator account for the large proportion of the fundamental closed loop is applied to achieve the goal of harmonic current reduction to construct harmonic control structure, as shown in figure 4.

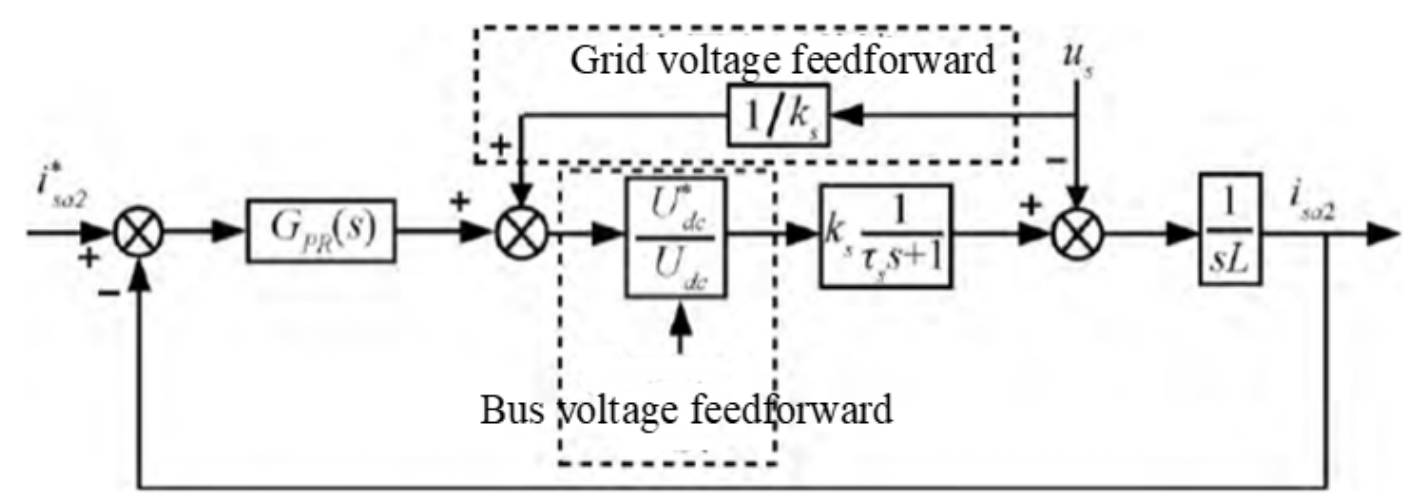

Fig. 4 dynamic control structure of aero-generator power supply closed-loop system based on current-loop resonant regulator

To sum up, in the current loop control system of aero-generator power supply, the electronic load equipment can be used to compensate the forward channel disturbance, and then the high-order 
harmonic disturbance can be analyzed again by using the grid voltage. Combined with figure 4, it ensures that both the voltage fluctuation of the power network and the influence of the inverter gain coefficient can satisfy the feedforward compensation control effect, and the disturbance of the current loop forward channel in the generator power supply is eliminated to the maximum.

On the other hand, it is necessary to observe the unit closed-loop feedback link of the inner loop network side of the power supply current, ensure that the feedback current and the instruction current are tracked accurately, and calculate all the current tracking amplitudes in the control current closed-loop system under the unit sinusoidal excitation effect. The current peak value is set from the position of the aero-generator power supply connected to the grid, the specific transmission coefficient of the DC side current is calculated, and the basic speed and the accuracy of the current measurement of the external loop regulation of the power supply voltage are improved in an all-round way [4].

\section{Conclusion}

The constant current electronic load is used to test the voltage source and current source, and it is even applied to the test process of the aero generator power supply. Finally, the relatively ideal simulation data are obtained, which is why the electronic load test is more preferable than the traditional manual test. It meets the requirements of feasibility, science and practicability of current voltage testing, and saves a lot of manpower, material resources and financial resources. It is worthy of extensive application and in-depth research.

\section{References}

[1] Song Bin. Application of electronic load in power supply test [J]. Journal of Weifang University.

[2] Cheng Fuping. Simulation study of energy-fed electronic load applied to power supply test [J]. Telecom Power Technologies 2015J: 107-108110.

[3] Zhang Yawei, Shi Qiangqiang, Yao Fenggang. Design of energy-fed electronic load in aeronautical power source test [J]. Foreign Electronic Measurement Technology

[4] Yang Xinxuan. Development of power electronic load for aero generator test [D]. Civil Aviation University of China, 2007. 